\title{
Phase-field modeling of dendritic growth of magnesium alloys with a parallel-adaptive mesh refinement algorithm
}

\author{
Bing-hui Tian', *Meng-wu Wu', Ang Zhang' ${ }^{2}$ Zhi-peng Guo ${ }^{3}$, Shou-mei Xiong ${ }^{3}$ \\ 1. Hubei Key Laboratory of Advanced Technology for Automotive Components, Wuhan University of Technology, Wuhan 430070, China \\ 2. National Engineering Research Center for Magnesium Alloys, Chongqing University, Chongqing 400044, China \\ 3. School of Materials Science and Engineering, Tsinghua University, Beijing 100084, China
}

\begin{abstract}
A two-dimensional phase field (PF) model was developed to simulate the dendritic solidification in magnesium alloy with hcp crystal structure. By applying a parallel-adaptive mesh refinement (Para-AMR) algorithm, the computational efficiency of the numerical model was greatly improved. Based on the PF model, a series of simulation cases were conducted and the results showed that the anisotropy coefficient and coupling coefficient had a great influence on the dendritic morphology of magnesium alloy. The dendritic growth kinetics was determined by the undercooling and equilibrium solute partition coefficient. A significant finding is acquired that with a large undercooling, the maximum solute concentration is located on both sides of the dendrite tip in the liquid, whereas the maximum solute concentration gradient is located right ahead of the dendrite tip in the liquid. The dendrite tip growth velocity decreases with the increase of the equilibrium solute partition coefficient, while the variation trend of the dendrite tip radius is the opposite. Quantitative analysis was carried out relating to the dendritic morphology and growth kinetics, and the simulated results are consistent with the theoretical models proposed in the previously published works.
\end{abstract}

Key words: magnesium alloy; dendritic growth; phase-field modeling; solidification

CLC numbers: TP391.9/TG146.22 Document code: A Article ID: 1672-6421 2021 06-541-09

\section{Introduction}

With the increasing demand for lightweight in automotive, aerospace, electronics and other fields, magnesium alloys have received widespread attention due to their advantages of low density, high specific strength, excellent castability and recyclability ${ }^{[1-3]}$. During alloy solidification, the dendritic growth is ubiquitous due to the instability of the solid-liquid interface ${ }^{[4,5]}$. Since the dendritic growth has a great influence on the phase transition, solute distribution and shrinkage formation during the solidification process, it plays a crucial role in determination of the solidification structure and final mechanical properties of castings ${ }^{[6-8]}$. So far, most of the published works relating to dendritic growth focused on alloys with face-centered cubic (fcc) and body-centered cubic (bcc)

\section{*Meng-wu Wu}

Male, born in 1984, Ph.D., Associate Professor. Research interests: advanced manufacturing technologies for automotive components.

E-mail:wumw@whut.edu.cn

Received: 2021-06-08; Accepted: 2021-07-21 crystal structures ${ }^{[9-13]}$, while very few studies focused on alloys with hexagonal close-packed (hcp) crystal structure. A consensus is reached that there are six preferred growth orientations of the dendrites in the basal plane (0001) for common magnesium alloys, that is, the dendrites grow along the direction $<11 \overline{2} 0>$ with a six-fold symmetry morphology in two dimensions under ideal solidification condition ${ }^{[14-16]}$. Since the practical solidification process usually involves with complicated fluid flow, heat transmission, solute diffusion, as well as the formation of porosity and second phase, the dendritic morphology of magnesium alloy is complex and diverse ${ }^{[17-19]}$. In other words, the complexity of intrinsic crystal structure and extrinsic solidification condition both lead to difficulties in studying the dendritic growth of magnesium alloy.

In recent years, numerical modeling and simulation has been rapidly developed as a powerful tool for predicting the time-dependent microstructure evolution during various solidification processes ${ }^{[20-22]}$. Among those numerical modeling techniques, both the cellular automation (CA) method and phase field (PF) method can effectively predict and depict the evolution of 
complex solidification structures, thus offer a deep insight into the dendritic growth during alloy solidification ${ }^{[23]}$. Particularly, with the development and application of adaptive mesh refinement (AMR) and parallel calculation algorithms, the computational efficiency of the PF model has been greatly improved. Therefore, the PF method has become a research hotspot in microstructure modeling and simulation for the present and even the future ${ }^{[24-26]}$.

Using the PF method, Wang et al. ${ }^{[27]}$ investigated the effects of thermal properties (thermal diffusivity and latent heat) on the morphological evolution of $\mathrm{Mg}$ during solidification, and the results showed that the thermal effects significantly influenced the shape evolution of the crystals and could control the formation of potential sites for void nucleation inside the crystal structures. Wang et al. ${ }^{[28]}$ coupled in situ synchrotron X-ray radiography and PF simulation to study the effect of cooling rate on dendritic morphology of $\mathrm{Mg}$ Gd alloys during directional solidification, and found that the simulated results were in good agreement with the experimental ones. Zhang et al. ${ }^{[29]}$ introduced the lattice Boltzmann method (LBM) into the PF model and studied the effect of stirring rate on the dendritic morphology of magnesium alloys, and the results indicated that fine and spherical grains could be obtained with a relative large stirring rate. Pan et al. ${ }^{[30]}$ used the PF method to simulate the dendritic growth of squeeze cast $\mathrm{Mg}$-Al alloy during the pressurized process, and found that the dendritic growth rate firstly increased and then decreased with the increase of pressure. By developing different anisotropy functions to describe the anisotropy of the solid-liquid interfacial energy, Yang et al. ${ }^{[31]}$, Wang et al. ${ }^{[32]}$ and Eiken ${ }^{[33]}$ established 3D PF models to simulate the dendritic growth of magnesium alloys in three dimensions. Through literature review, though there are some simulation works reported on the dendritic growth of magnesium alloys based on PF method, little attention has been paid to the dendritic growth kinetics of magnesium alloys. Meanwhile, the current research is lack of quantitative analysis relating to the effect of parameters in the PF model on the dendritic morphology and growth kinetics of magnesium alloys.

In the present work, a PF model was developed to simulate the dendritic growth of magnesium alloy coupling with a Para-AMR algorithm. Case studies were conducted to verify the computing accuracy and efficiency of the numerical model. The influence of different phase field parameters and solidification process conditions on the dendritic morphology and growth kinetics of magnesium alloy was studied. The relevant simulated results were compared with those in the previously published works.

\section{Numerical model}

\subsection{Phase field model}

A PF model coupled with thermal-solute transport proposed by Ramirez et al. ${ }^{[34]}$ was adopted. The total free energy $F$ of the system can be expressed as:

$$
F[\phi, c, T]=\int\left[\frac{\sigma}{2}|\nabla \phi|^{2}+f_{A B}(\phi, c, T)\right] d V
$$

where $\phi$ is the phase field which varies smoothly from -1 in liquid to 1 in solid, $c$ and $T$ are the actual solute concentration and temperature of the liquid, respectively, $\sigma$ is a gradient energy coefficient, $f_{A B}(\phi, c, T)$ is the bulk free energy density, $V$ is the total volume of the system. The governing equations of the phase field model determined by the variation form of the free-energy function can be written as:

$$
\begin{gathered}
\tau_{0} \frac{\partial \phi}{\partial t}=-K_{\phi} \frac{\delta F}{\delta \phi} \\
\frac{\partial c}{\partial t}=\nabla \cdot\left(K_{\mathrm{C}} \nabla \frac{\delta F}{\delta c}-\boldsymbol{j}_{\mathrm{at}}\right)
\end{gathered}
$$

where $t$ is the time, $\tau_{0}$ is the relaxtion time, $K_{\phi}$ and $K_{\mathrm{C}}$ are constant, $\boldsymbol{j}_{\mathrm{at}}$ is an anti-trapping current to eliminate non-equilibrium effects at the diffuse interface, which is given by:

$$
\boldsymbol{j}_{\mathrm{at}}=-\frac{1+(1-k) U}{2 \sqrt{2}} \frac{\partial \phi}{\partial t} \frac{\nabla \phi}{|\nabla \phi|}
$$

where $k$ is the equilibrium solute partition coefficient, $U$ is the dimensionless solute concentration. The governing equations for phase field (crystal anisotropy) can be finally written as:

$$
\begin{gathered}
A(\boldsymbol{n})^{2}\left[\frac{1}{L e}+k(1+(1-k) U)\right] \frac{\partial \phi}{\partial t} \\
=\frac{1}{2} \nabla \cdot\left[\frac{\partial A(\boldsymbol{n})^{2}|\nabla \phi|^{2}}{\partial \nabla \phi}\right]+\phi-\phi^{3}-\lambda\left(1-\phi^{2}\right)^{2}(\theta+k U) \\
\frac{1+k-(1-k) \phi}{2} \frac{\partial U}{\partial t}=\nabla \cdot\left[D \frac{1-\phi}{2} \nabla U-\boldsymbol{j}_{\text {at }}\right]+\frac{1+(1-k) U}{2} \frac{\partial \phi}{\partial t}(6)
\end{gathered}
$$

where $L e$ is the Lewis number, i.e., $L e=\alpha / D, \alpha$ and $D$ are thermal and solute diffusivities, respectively. The value of $L e$ was chosen to be 1,000 in this study. $A(\boldsymbol{n})$ is the anisotropy function, and $\boldsymbol{n}$ denotes the unit vector normal to the solid-liquid interface. For the crystal with six-fold symmetry morphology, $A(\boldsymbol{n})$ is given by ${ }^{[35]}$.

$$
A(\boldsymbol{n})=1+\varepsilon \cos (6 \varphi)
$$

where $\varepsilon$ is the anisotropy coefficient and $\varphi$ denotes the angle between the solid-liquid interface norm $\boldsymbol{n}$ and the axis $x, \lambda$ is the coupling coefficient, which can be expressed as:

$$
\lambda=\frac{15 R_{\mathrm{g}} T_{\mathrm{M}}(1-k)}{16 V_{\mathrm{mol}} H|m|} \Delta T_{0}
$$

where $R_{\mathrm{g}}$ is the gas constant, $T_{\mathrm{M}}$ is the melting temperature of the alloy, $V_{\text {mol }}$ represents the molar volume, $H$ denotes the dimensionless energy barrier of the double well potential, $m$ is the liquidus slope and $\Delta T_{0}$ denotes the equilibrium freezing temperature range. The dimensionless solute concentration $U$ and 
undercooling $\theta$ are given by

$$
\begin{gathered}
U=\frac{\frac{2 c / c_{\infty}}{1+k-(1-k) \phi}-1}{1-k} \\
\theta=\frac{T-T_{\mathrm{M}}-m c_{\infty}}{\Delta T_{0}}
\end{gathered}
$$

where $c_{\infty}$ is the initial solute concentration of the liquid. The length and time are scaled by $W_{0}=\lambda d_{0} / a_{1}$ and $\tau_{0}=d_{0}^{2} a_{2} \lambda^{3} /\left(D a_{1}^{2}\right)$ from the real to the unit of PF model, respectively, where $d_{0}=\Gamma / \Delta T_{0}$ is the chemical capillary length, $\Gamma$ is the GibbsThomson coefficient, $a_{1}=0.8839$ and $a_{2}=0.6267^{[36]}$.

\subsection{Para-AMR algorithm}

A Para-AMR algorithm developed by Guo et al. ${ }^{[37,38]}$ was applied to improve the computing efficiency of the PF model. The algorithm consists of two parts: adaptive meshing and parallel computing. The first step of adaptive meshing is to find the grids that need to be coarsened or refined according to a gradient criterion:

$$
|\nabla \phi|+\beta_{U}|\nabla U|+\beta_{\theta}|\nabla \theta| \geq \xi
$$

where $\beta_{U}, \beta_{\theta}$ are the weight coefficients for the dimensionless solute concentration and undercooling, respectively, $\beta_{U}=0.3$ and $\beta_{\theta}=0 . \xi$ is a threshold value which has to be retrieved via numerical tests. With the gradient criterion, the solid-liquid interface grids, where the phase transition is the fastest and precise characterization is the most required, could be marked. After certain grids/points have been tagged, a cluster algorithm was adopted to separate the tagged grids into patch-boxes at each level, resulting in a data structure with a clear hierarchical configuration. The values of the overlapped regions were then updated through interpolation and restriction operations.

To reduce the computation overhead, the parallel computing method was employed and integrated into the AMR algorithm. After generating the adaptive grids, the layout of the patchboxes including all information regarding to the mesh configuration was broadcasted to all processors to realize parallel computing. As a result, each processor would have its own but different arrays of patch-boxes. The data with respect to phase field, solute field and temperature field would then be constructed accordingly on each processor, achieving the socalled single program with multiple data ${ }^{[38]}$.

\subsection{Accuracy and efficiency tests}

Simulation cases with different grid levels were conducted to verify the accuracy of the numerical model. One solid cell was initially seeded in the middle of the calculation domain. A uniform grid size ( $N_{\mathrm{amr}}=1, N_{\mathrm{amr}}$ is the number of the grid levels employed in the simulation) of $\mathrm{d} x=0.8$ was employed, the total grid number of the domain was $2,048 \times 2,048$. For non-uniform grids, the grid size at the top grid level was set to be $\mathrm{d} x_{\min }=0.8$. An increase of the bottom grid size would lead to a decrease of the bottom grid number, i.e., when $N_{\mathrm{amr}}=2$ and $\mathrm{d} x=1.6$, the bottom grid number was $1,024 \times 1,024$, when $N_{\mathrm{amr}}=3$ and

$\mathrm{d} x=3.2$, the bottom grid number was $512 \times 512$.

Figure 1 shows the simulated dendrite tip morphology with different grid levels. As illustrated in Fig. 1(a), all grids are of the same size, including the bulk grids in the liquid phase region far away from the solid-liquid interface. It can be believed that it wastes of computing resources since the bulk grids are not involved in phase transition and thermal-solute diffusion. As for $N_{\text {amr }}=2$ and $N_{\text {amr }}=3$ [Figs. 1(b, c)], the grid size near the solid-liquid interface is the same as the grid size when $N_{\mathrm{amr}}=1$. For the liquid phase region far away from the solidification interface, the larger the $N_{\mathrm{amr}}$, the larger the grid size and the smaller the number of grids. Therefore, increasing the number of the grid levels, $N_{\text {amr }}$, is of great benefit to saving computing resources. Although different meshing strategies are adopted, it can be seen from Fig. 1(d) that there are slight differences among the dendrite tip outlines, which can be considered negligible. Consequently, the accuracy of the PF model coupled with the AMR algorithm was verified.
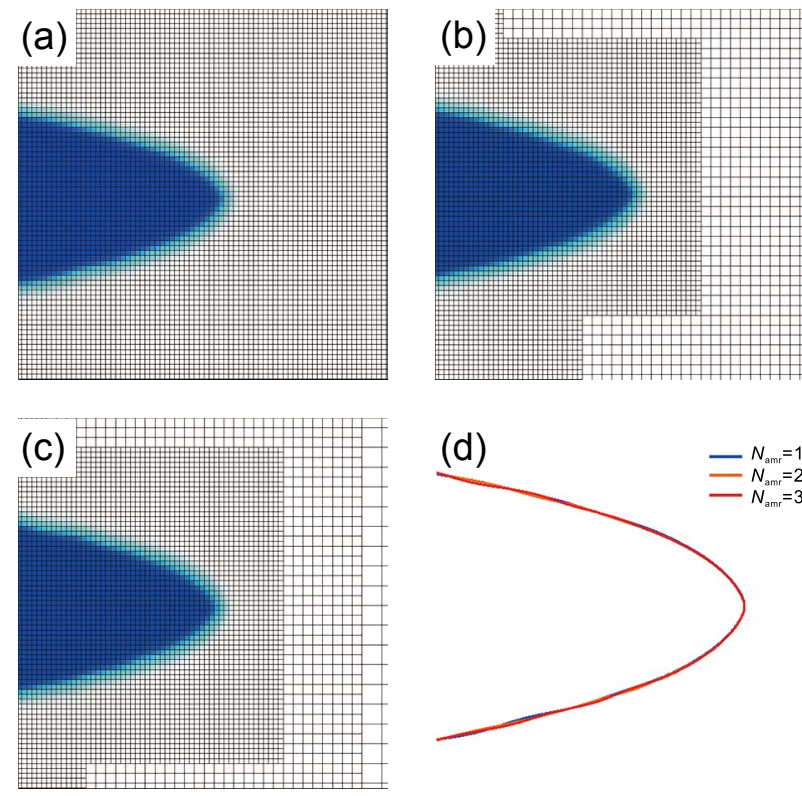

Fig. 1: Simulated dendrite tip morphology with different grid levels: (a) $N_{\mathrm{amr}}=1$; (b) $\boldsymbol{N}_{\mathrm{amr}}=2$; (c) $N_{\mathrm{amr}}=3$; (d) dendrite tip outlines retrieved according to $\Phi=0.9$ with different $\boldsymbol{N}_{\mathrm{amr}}$

The efficiency of the Para-AMR algorithm was tested by changing the number of $N_{\mathrm{amr}}$ and $N_{\mathrm{p}}$, where $N_{\mathrm{p}}$ is the number of the parallel processors. In all simulation cases, one solid cell was initially seeded in the middle of the calculation domain and the top grid level was set to be $\mathrm{d} x_{\min }=0.8$. Figure 2 shows the efficiency test results, in which each line represents a serial of simulation cases with the same calculation domain and termination step. For instance, the elapsed time counted in all simulation cases in line $\mathrm{L}_{1}$ is the time from starting the program until running to the termination step of 3,500 . Meanwhile, since the adaptive meshing technique was adopted, the domain size $819.2 \times 819.2$ was just equal to a domain size consisting of $1,024 \times 1,024$ grids with a uniform grid size of $\mathrm{d} x=0.8$. Here, the domain size $1,638.4 \times 1,638.4$ was four times as large as $819.2 \times 819.2$. 
(a)

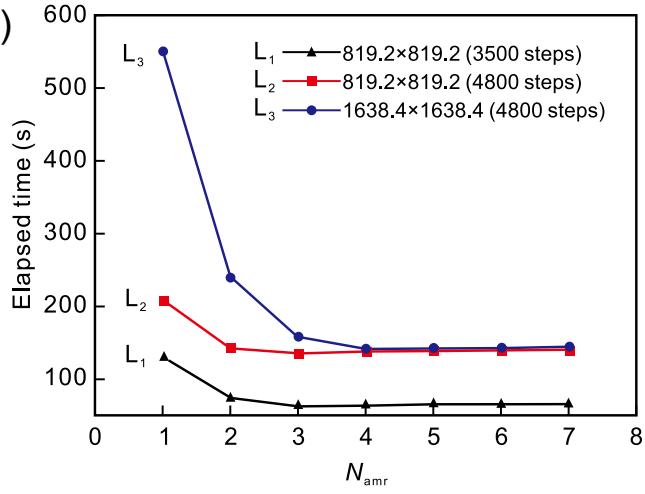

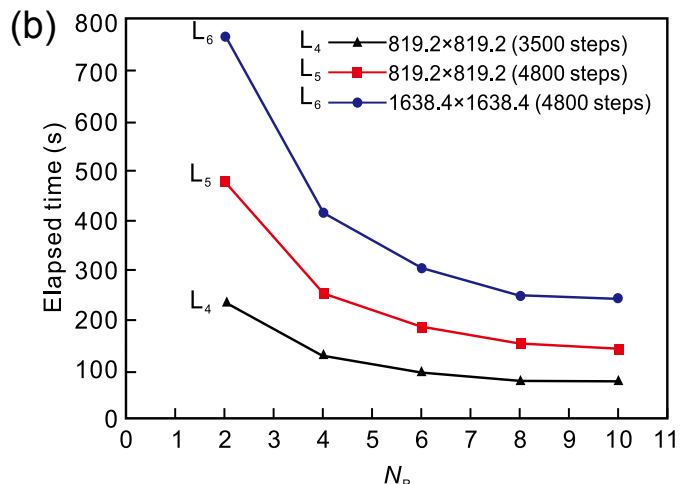

Fig. 2: Efficiency test of Para-AMR algorithm: (a) elapsed time versus $N_{\text {amr, }}$ while in all simulation cases $N_{\mathrm{p}}=10$; (b) elapsed time versus $N_{\mathrm{p}}$, while in all simulation cases $N_{\mathrm{amr}}=2$

As shown in Fig. 2(a), the elapsed time decreases with the increase of $N_{\text {amr }}$. For the simulation cases in line $\mathrm{L}_{3}$, the application of the AMR algorithm with $N_{\text {amr }}=4$ shortens the total elapsed time from $552 \mathrm{~s}$ to $141 \mathrm{~s}$, demonstrating excellent ability of the algorithm to improve the computational efficiency. Comparing lines $\mathrm{L}_{1}$ and $\mathrm{L}_{3}$, it can be found that the advantage of the AMR algorithm is more obvious with a greater calculation scale. As for lines $\mathrm{L}_{2}$ and $\mathrm{L}_{3}$ with different calculation domains, the elapsed time in line $\mathrm{L}_{3}$ is much longer than that in line $\mathrm{L}_{2}$ when $N_{\mathrm{amr}}=1$ (without AMR). However, this situation gradually changes with the increase of $N_{\text {amr }}$ and the elapsed time in two lines tends to be the same. This is because when $N_{\text {amr }}$ is small, the proportion of the number of the grids far away from the solid-liquid interface is relatively large, resulting in waste of lots of computing resources. With the increase of $N_{\text {amr }}$, the grids far away from the solid-liquid interface are coarsened, and the proportion of the number of these grids is reduced, thereby saving computing resources and improving the computational efficiency. It can be seen from Fig. 2(b) that by applying the parallel computing technique, the computational efficiency of the numerical model is also greatly improved. With the increase of $N_{\mathrm{p}}$, the elapsed time of the simulation cases decreases. And the larger the calculation scale, the greater the improvement in computational efficiency. For example, the total elapsed time in line $\mathrm{L}_{6}$ shortens from $761 \mathrm{~s}$ when $N_{\mathrm{p}}=2$ to $239 \mathrm{~s}$ when $N_{\mathrm{p}}=10$.

It is worth mentioning that the elapsed time does not always decrease as $N_{\text {amr }}$ increases. As illustrated in Fig. 2(a), when $N_{\text {amr }}$ reaches a certain value, i.e., $N_{\text {amr }}>4$ in line $\mathrm{L}_{3}$ or $N_{\text {amr }}>3$ in line $\mathrm{L}_{2}$, the elapsed time increases slightly. This is because when a larger value of $N_{\mathrm{amr}}$ is adopted, a more complicated hierarchical architecture would increase the cost of regridding process. In such case, the computational efficiency of the numerical model is not improved as expected. At the same time, as indicated in Fig. 2(b), when $N_{\mathrm{p}}$ was large enough, its improvement in computational efficiency is very limited with the increase of $N_{\mathrm{p}}$. The reason can be attributed to an increment of the cost of data communication process among the parallel processors. Therefore, appropriate values of $N_{\text {amr }}$ and $N_{\mathrm{p}}$ should be chosen for a specific simulation case with a certain calculation scale.

\section{Simulation results and discussion}

\subsection{Effect of anisotropy coefficient $(\varepsilon)$ on dendritic morphology}

The anisotropy coefficient $(\varepsilon)$ indicates the anisotropy degree of surface tension, thickness and dynamics of the interface. To explore the effect of $\varepsilon$ on the dendritic morphology of magnesium alloy, phase field simulations of a single dendrite growth were performed by planting a solid seed in the middle of the calculation domain. Key parameters used in the simulations were set as $\theta=0.25, k=0.15$ and $\lambda=10$. Figure 3 shows the simulated dendritic morphology of magnesium alloy with different values of $\varepsilon$. The dendritic morphology is approximately square when $\varepsilon=0$ as illustrated in Fig. 3(a). There are eight primary branches symmetrically distributed, and the adjacent primary branches are parallel to each other (e.g. the branches A and B) or formed an angle of $90^{\circ}$ (e.g. the branches B and C). With the increase of $\varepsilon$, the preferred growth orientations of the dendrite change. As shown in Fig. 3(b), two more primary branches marked $\mathrm{D}$ and $\mathrm{E}$ appear along the horizontal direction compared with the dendritic morphology when $\varepsilon=0$. Furthermore, the angle between the branches $\mathrm{D}$ and $\mathrm{F}$ and $\mathrm{F}$ and $\mathrm{G}$ is $60^{\circ}$. It can be seen from Figs. 3(c, d) that with a greater value of $\varepsilon$, the sixfold symmetry characteristic of the dendritic morphology is more obvious. Therefore, with the increase of $\varepsilon$, the dendritic morphology of magnesium alloy changes from a square into a seaweed, and finally a typical six-fold symmetry shape.

\subsection{Effect of undercooling $(\theta)$ and coupling coefficient $(\lambda)$ on dendritic morphology}

According to the classical solidification theory, the undercooling $(\theta)$ is the driving force for solidification of liquid metals ${ }^{[39]}$. In the PF model, the couple coefficient $(\lambda)$ is an important parameter reflecting the relationship between heat and solidification. As expressed in Eq. (8), $\lambda$ is determined by the physical parameters of the alloy, however, the exact values of some of them are difficult to obtain at the moment. Consequently, the effect of $\theta$ and $\lambda$ on the dendritic morphology of magnesium alloy was studied by the present PF model. During the simulations, the anisotropy coefficient and equilibrium solute partition coefficient were set as $\varepsilon=0.02$ 


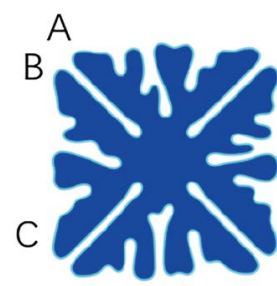

(a)

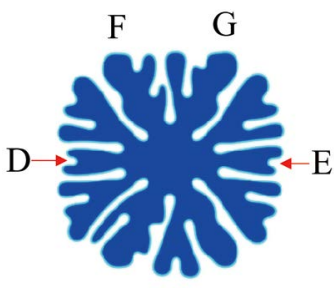

(b)

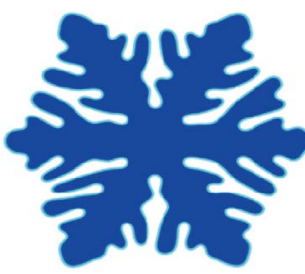

(c)

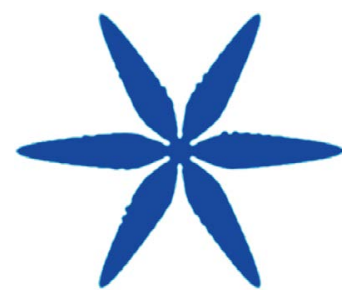

(d)

Fig. 3: Simulated dendritic morphology of magnesium alloy with different anisotropy coefficients $(\varepsilon)$ : (a) 0 ; (b) 0.001 ; (c) 0.002 ; (d) 0.02

and $k=0.15$. It can be seen from Fig. 4 that the vaules of $\theta$ and $\lambda$ have a great influence on the dendritic morphology of magnesium alloy. Taking $\lambda=10$ as an example, with the increase of $\theta$, the dendrite grows into a more well-developed structure. Only primary branches are observed when $\theta=0.22$ and 0.25 [Figs. 4(a,b)], while a few secondary branches bifurcate on the primary branches when $\theta=0.28$ [Fig. 4(c)]. The secondary branches develope sufficiently when $\theta=0.30$, and even some tertiary branches are observed bifurcating on the secondary branches [Fig. 4(d)]. This phenomenon can be explained as follows: with the increase of $\theta$, the driving force for solidification and denritic growth velocity of magnesium alloy increases, resulting in a reduciton of the stability of solidification interface. In this case, the primary branches of the dendrite become slender, and the disturbance at the solidification interface will induce the formation of secondary, tertiary and even higher-order branches.

As for $\lambda$, its effect on the dendritic morphology of magnesium alloy is the same as that of $\theta$. Taking $\theta=0.25$ for instance, it can be seen that the dendrite is well-developed with the increase of $\lambda$ [Figs. 4(b, f)]. Moreover, the horizontal right primary branch is splitted into two branches when $\lambda=34$ as shown in Fig. 4(j). This phenomenon is more obvious when $\lambda$ increases to 40 , while bifurcation structures form at the locations occupied by the original horizontal left and right primary branches [Fig. 4(n)].

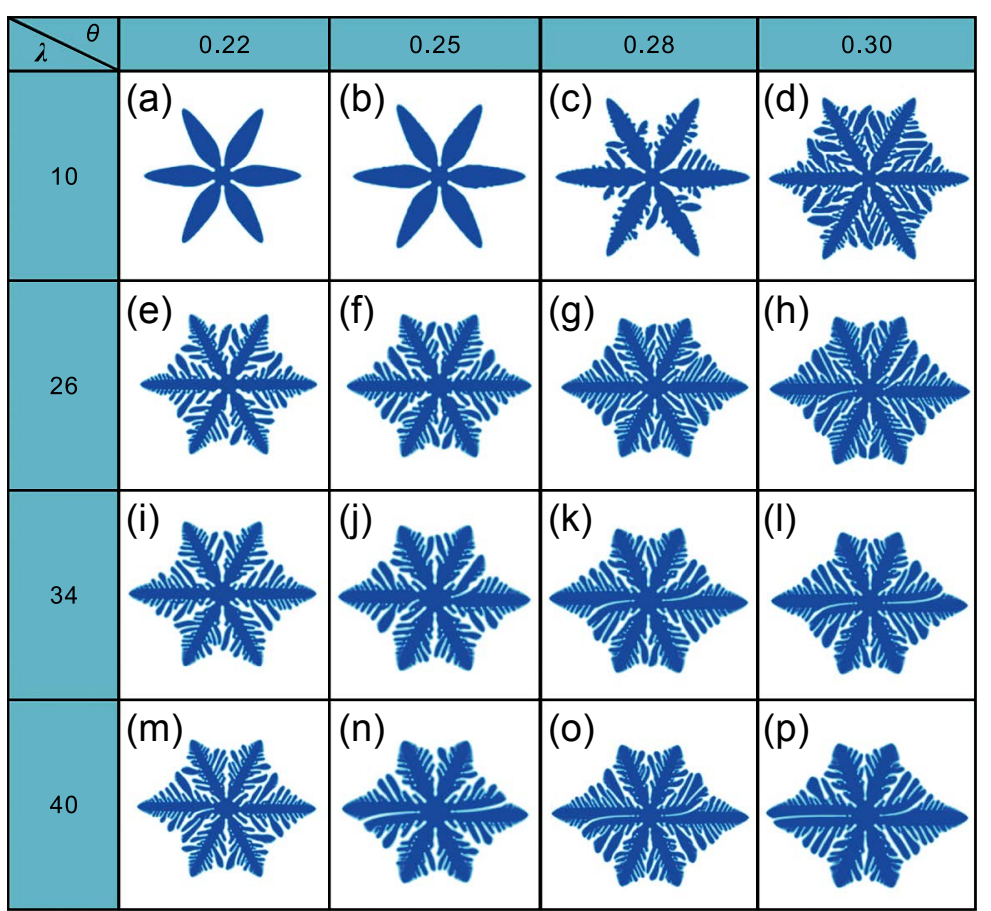

Fig. 4: Simulated dendritic morphology of magnesium alloy with different undercooling $(\theta)$ and coupling coefficients $(\lambda)$
A conclusion can be made that the larger the $\lambda$, the more unstable the solidification interface and the more complex the dendritic morphology of magnesium alloy. What is particularly interesting about the bifurcation structure is that it can form when a small $\theta$ is employed with a large $\lambda$, or a small $\lambda$ is employed with a large $\theta$. For example, the bifurcation structure is observed both in Fig. 4(m) when $\lambda=40, \theta=0.22$ and Fig. 4(h) when $\lambda=26, \theta=0.30$. Therefore, the dendritic morphology of magnesium alloy is affected by both the inherent factors (such as $\lambda$ while it is determined by the physical parameters of the alloy) and external factors (such as $\theta$ ) during the solidification process.

\subsection{Effect of undercooling $(\theta)$ and equilibrium solute partition coefficient $(k)$ on dendritic growth kinetics}

The growth behavior of the dendrite tip has a great influence on the dendritic morphology and growth kinetics of magnesium alloy. Based on the present PF model, the detailed characteristics of the dendrite tip was studied. Figure 5 shows the simulated dendrite tip morphology with different undercooling $(\theta)$. Key parameters used in the simulations were set as $\varepsilon=0.02, \lambda=10$ and $k=0.15$. The solid outlines of dendrite tip were retrieved according to $\phi=0.9$. It can be found that with the increase of $\theta$, the dendrite tip becomes more slender, resulting in a reduction of the dendrite tip radius $(R)$. Dotted parabolas are used to fit the solid outlines of the dendrite tip, it can be seen that they are in good agreement with each other. Hence, the PF simulations visually confirmed the statement in classsical solidification theory with regard to the dendrite tip morphology ${ }^{[40]}$.

Further modeling studies were conducted to quantitatively investigate the effect of $\theta$ on the dendritic growth kinetics of magnesium alloy. As illustrated in Fig. 6(a), $R$ decreases rapidly with the increase of $\theta$ in the beginning. Then the decrease slows down, and there seems to be a minimum 

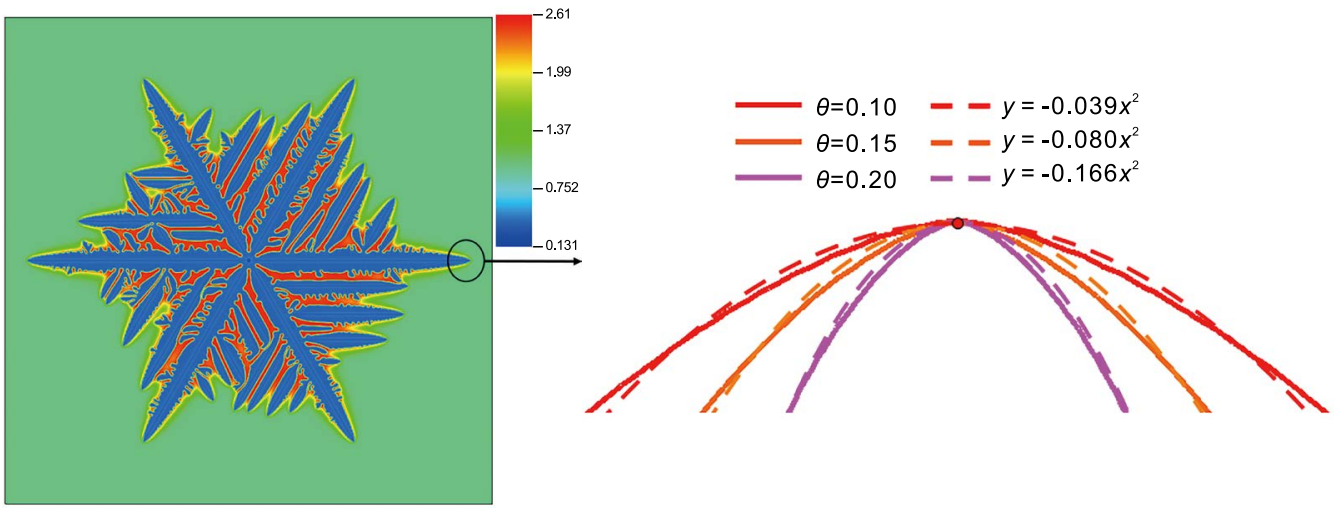

Fig. 5: Simulated dendrite tip morphology with different undercooling $(\theta)$, while the solid outlines of dendrite tip were retrieved according to $\Phi=0.9$ and the dotted parabolas were used for fitting

(a)

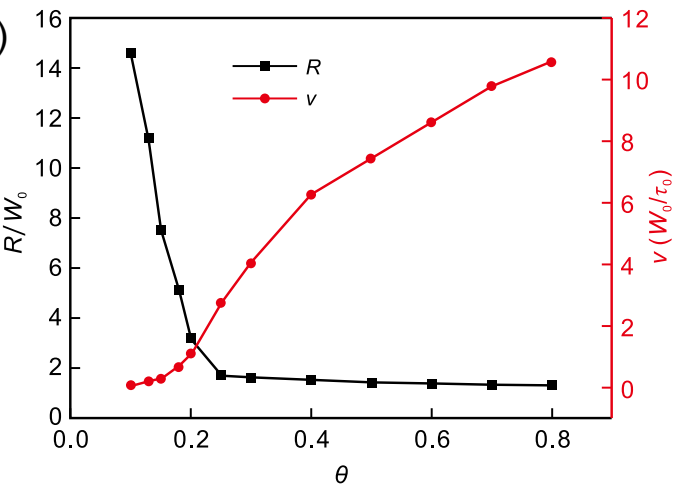

(b)

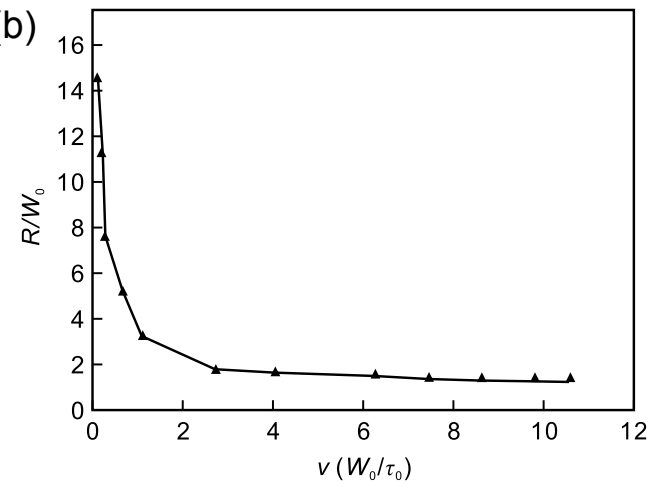

Fig. 6: Effect of undercooling on dendritic growth kinetics: (a) dendrite tip radius $(R)$ and growth velocity $(v)$ versus undercooling $(\theta)$; $(b)$ dendrite tip radius versus growth velocity

value of $R$ when $\theta$ is large enough. Kurz et al. ${ }^{[41]}$ considered that the minimum value of $R$ was just euqal to the critical nucleus radius. With respect to the relationship between the dendrite tip growth velocity $(v)$ and $\theta$, the driving force for solidification is heightened with the increase of $\theta$, thereby $v$ increases. It can be found that the increment experienced a history of slow-fast-slow rate, which was consistent with the LGK model ${ }^{[42]}$. As indicated in Fig. 6(b), $R$ is almost in inverse proportion to $v$, while the simulated results are in accordance with the theoretical model proposed by Kurz et al ${ }^{[41]}$.

Strictly speaking, the undercooling mentioned above was the thermal undercooling. During alloy solidification, the solute distribution also has a great impact on the dendritic growth kinetics. Figure 7 shows the simulated solute distribution at the dendrite tip of the magnesium alloy with different $\theta$. As the solidification proceeds, there is solute rejection in front of the solidification interface. Furthermore, the solute in the liquid metal diffuses according to the Fick's second law. Comparing Fig. 7(a) with Fig. 7(b), it can be seen that with a larger $\theta$, the dendrite tip grows faster, resulting in an insufficient diffusion of the solute in front of the solidification interface. A significant finding is acquired in Figs. 7(b, d) that the maximum solute concentration is unexpectedly located on both sides of the dendrite tip in the liquid, whereas the maximum solute concentration gradient is located right ahead of the dendrite tip in the liquid. The solute accumulation in front of the solidification interface would cause the so-called constitutional undercooling to form in the liquid metal, which has a negative effect on the total undercooling. This is just the reason why the increment of $v$ experiences a history of slow-fast-slow rate with the increase of $\theta$ mentioned above. Meanwhile, a high accumulation of the solute on both sides of the dendrite tip may lead to lateral branches bifurcated on the trunk, as shown in Fig. 7(b).

Taking a deeper look at the solute distribution in front of the solidification interface and the dendritic grow kinetics, the equilibrium solute partition coefficient $(k)$ plays an important role in them. Figure 8 shows the simulated dendritic morphology of magnesium alloy and solute distribution with different $k$. During the simulations, key parameters were set as $\varepsilon=0.02$, $\theta=0.25$ and $\lambda=10$. It can be found from Figs. $8(\mathrm{a}, \mathrm{b})$ that with a lower $k$, a higher accumulation of the solute is observed in front of the solidification interface, leading to a more well-developed dendritic structure. The qualitative analysis is well verified by quantitative statistics of the solute concentration along the horizontal direction of the dendrite tip, as illustrated in Fig. 8(c). This can be exactly explained according to the definition of $k$. In the definition, local equilibrium at the solid-liquid interface is assumed, then an expression can be acquired as $c_{\mathrm{S}}^{*}=k c_{\mathrm{L}}^{*}$, where $c_{\mathrm{S}}^{*}$ and $c_{\mathrm{L}}^{*}$ are the equilibrium solute concentrations of the solid and liquid phases at the interface, respectively. As the liquid phase transforms into the solid phase, the solute rejected into the adjacent liquid is $\Delta c=c_{\mathrm{L}}^{*}-c_{\mathrm{S}}^{*}=(1-k) c_{\mathrm{L}}^{*}$. In this case, a lower $k$ would undoubtedly lead to a higher accumulation of the solute 

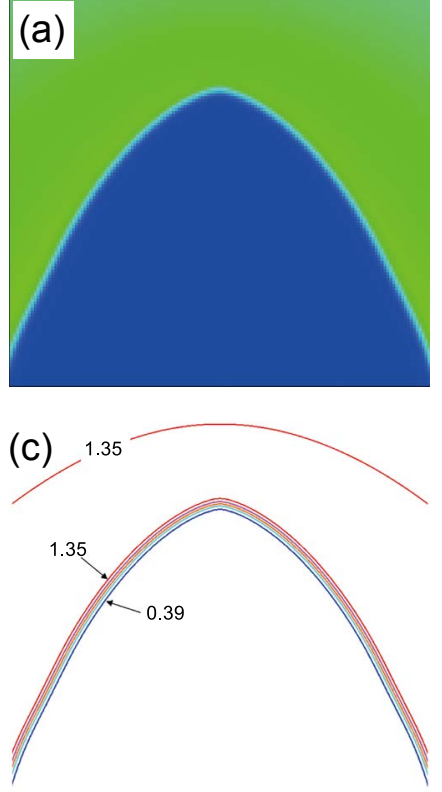
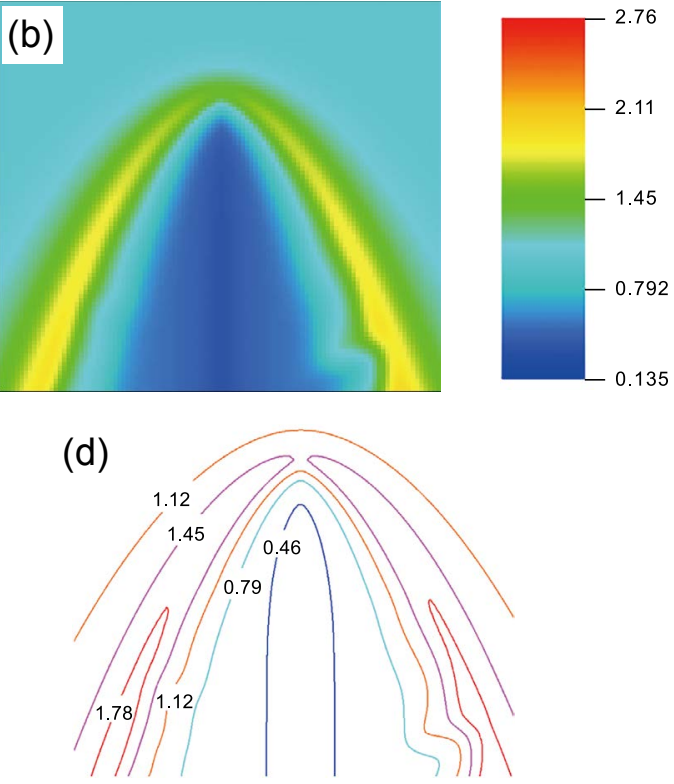

Fig. 7: Solute distribution at the dendrite tip with different undercooling $(\theta)$ : (a) Pseudo-color plot, $\theta=0.13$; (b) Pseudo-color plot, $\theta=0.30$; (c) contour plot, $\theta=0.13$; (d) contour plot, $\theta=0.30$
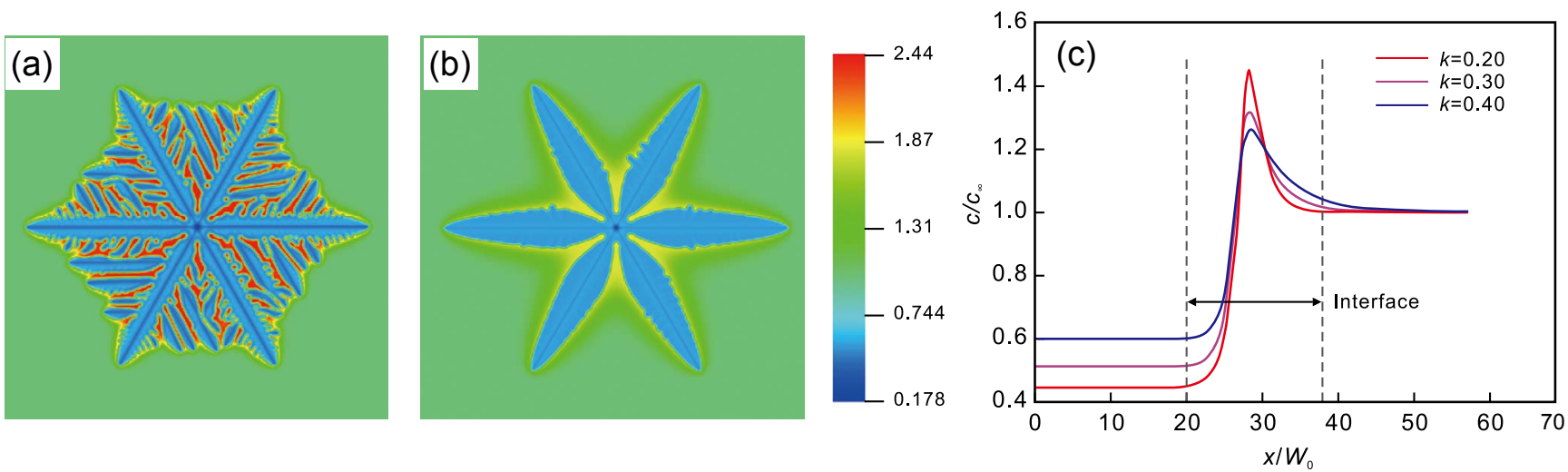

Fig. 8: Simulated dendritic morphology and solute distribution with different equilibrium solute partition coefficients (k): (a) $k=0.2$; (b) $k=0.3$; (c) solute distribution curves along horizontal direction of dendrite tip

in front of the solidification interface. Meanwhile, the solute concentration of the solid phase increases with the increase of $k$ as shown at the left end of the curves in Fig. 8(c). And at the right end of the curves, the solute concentration of the liquid phase with different $k$ tends to be the same, i.e., the initial solute concentration of the liquid $\left(c_{\infty}\right)$.

Figure 9 shows the simulated results relating to the relationship among the dendrite tip growth velocity $(v)$, radius $(R)$ and equilibrium solute partition coefficient $(k)$. It can be seen that $v$ decreases with the increase of $k$, while the variation trend of $R$ is the opposite. Moreover, regardless of the value of $k, R^{2} v k$ is almost equal to a constant value, i.e., $R^{2} v k \approx 2.02$ in the present work (the dotted line was obtained by fitting the data points of $R^{2} v k$ ). The simulated results are consistent with the theoretical model proposed by Kurz et al ${ }^{[41]}$, which could be expressed as $R^{2} v k=4 \pi^{2} D \Gamma / \Delta T_{0}$. To some extent, the values of some physical parameters of metal alloys, such as $D$ and $\Gamma$ maybe acquired by the method of modeling and simulation in the way mentioned above, while they are difficult to be obtained by experiments.

\subsection{Competitive growth of two equiaxed dendrites}

The practical solidification process usually involves a number of crystal nucleation and dendritic growth. In this case, there exists competitive growth among multiple dendrites, which inevitably has an important influence on the solute distribution and growth morphology of dendrites. In order to explore the influence law, a simulation case was carried out while two nuclei were set in the calculation domain. Key parameters used in the simulation were set as $\varepsilon=0.02, \lambda=10, k=0.15$ and $\theta=0.26$. It can be seen from Fig. 10(a) that the two dendrites grow independently in the early stage of solidification. As the solidification proceeds, the dendrites gradually impinge on each other, which results in coarsening of the dendrite tips as shown in Fig. 10(b).

As for the solute distribution, it can be found in Fig. 10 (c) that the solute fields of the two dendrites do not interfere with each other in the early stage of solidification until the solidification proceeds up to 6,000 steps in the PF 


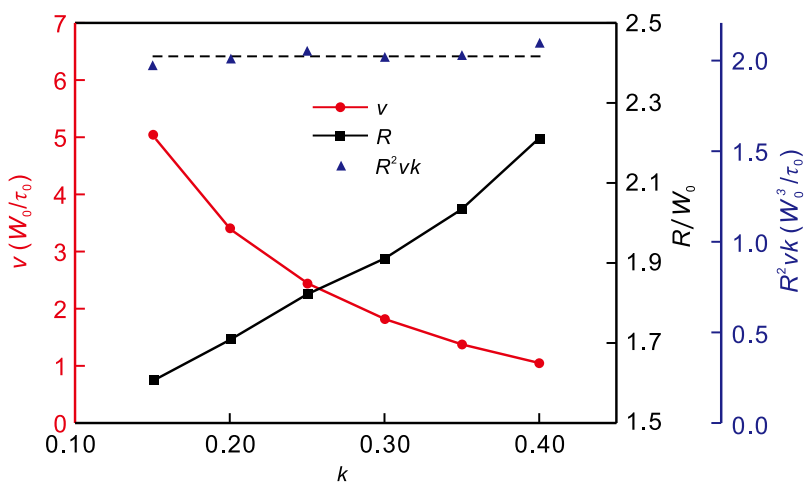

Fig. 9: Simulated results relating to the relationship among the dendrite tip growth velocity, radius and equilibrium solute partition coefficient simulation. During this period, the solute concentration in the liquid at the dendrite tip keeps an enrichment degree of 1.48. As the solidification proceeds over 6,000 steps, there is a superposition effect between the solute fields of the two dendrites. In this case, the enrichment degree mentioned above gradually increases, and reaches a peak value of 1.83 at the step of 6,400 . It is worth mentioning that when the soldification continues to proceed, the enrichment degree of the solute concentration in the liquid between the two dendrite tips with opposite directions decreases. For example, this value decreased to 1.69 at the step of 6,500 . The reason was that the two dendrite tips grew slower and slower once they impinged on each other, thus the soulte rejected into the
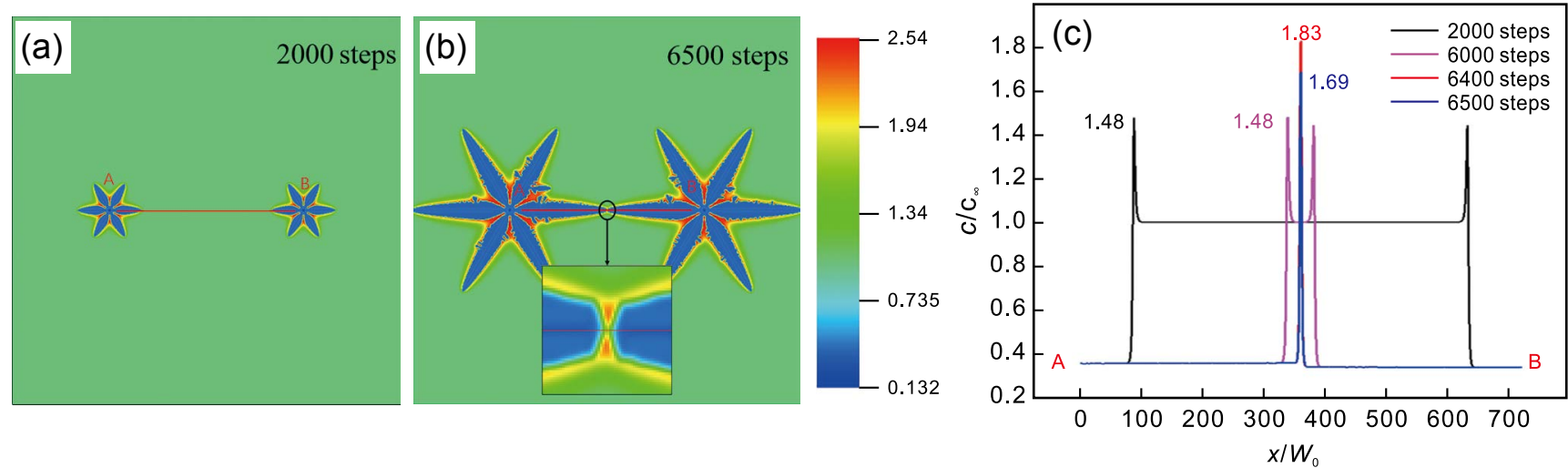

Fig. 10: Simulated competitive growth of two equiaxed dendrites of magnesium alloy: (a) 2,000 steps;

(b) 6,500 steps; (c) solute distribution along the line A-B

liquid was greatly reduced. Then, the enrichment degree of solute concentration will decrease as soon as the diffusion rate exceeds the accumulation rate of solute.

\section{Conclusions}

(1) The computational efficiency of the PF model coupled with a Para-AMR algorithm is greatly improved by appropriately increasing the numbers of the grid levels and the parallel processors.

(2) An increase of the undercooling and coupling coefficient both lead to a more well-developed dendritic structure of magnesium alloy. When they are large enough, bifurcation structures will form, resulting in a more complex dendritic morphology of magnesium alloy.

(3) With a large undercooling, the maximum solute concentration is located on both sides of the dendrite tip in the liquid, whereas the maximum solute concentration gradient is located right ahead of the dendrite tip in the liquid. The dendrite tip growth velocity decreases with the increase of the equilibrium solute partition coefficient, while $R^{2} v k$ is almost equal to a constant value.

(4) The simulated results relating to the dendritic morphology and growth kinetics of magnesium alloy are in good agreement with the theoretical models proposed by Kurz et al.

\section{Acknowledgements}

This work was financially supported by the National Natural Science Foundation of China (No. 51805389), the Natural Science Foundation of Hubei Province, China (No. 2018CFB210) and the 111 Project (No. B17034).

\section{References}

[1] Song J F, She J, Chen D L, et al. Latest research advances on magnesium and magnesium alloys worldwide. Journal of Magnesium and Alloys, 2020, 8(1): 1-41.

[2] Wang $X J, X u D K, W u R Z$, et al. What is going on in magnesium alloys? Journal of Materials Science and Technology, 2018, 34(2): 245-247.

[3] You Z Y, Jiang A X, Duan Z Z, et al. Effect of heat treatment on microstructure and properties of semi-solid squeeze casting AZ91D. China Foundry, 2020, 17(3): 219-226.

[4] Wang $Y$, Xing S M, Ao X H, et al. Microstructure evolution of A380 aluminum alloy during rheological process under applied pressure. China Foundry, 2019, 16(6): 371-379.

[5] Huang S C, Glicksman M E. Fundamentals of dendritic solidification-I. Steady state tip growth. Acta Metallurgica, 1981, 29(5): 701-715.

[6] Asta M, Beckermann C, Karma A, et al. Solidification microstructures and solid-state parallels: Recent developments, future directions. Acta Materialia, 2009, 57(4): 941-971. 
[7] Guo K Y, Xu C, Lin X P, et al. Microstructure and strengthening mechanism of Mg-5.88Zn-0.53Cu-0.16Zr alloy solidified under high pressure. Transactions of Nonferrous Metals Society of China, 2020, 30(1): 99-109.

[8] Zhou J X, Wang J, Wang J, et al. Effects of RE and Sr additions on dendrite growth and phase precipitation in AZ91D magnesium alloy. Transactions of Nonferrous Metals Society of China, 2010, 20(s2): s331-s335.

[9] Lee J, Ohno M, Shibuta Y, et al. Uniquely selected primary dendrite arm spacing during competitive growth of columnar grains in Al-Cu alloy. Journal of Crystal Growth, 2021, 558: 126014.

[10] Becker M, Dantzig J A, Kolbe M, et al. Dendrite orientation transition in Al-Ge alloys. Acta Materialia, 2019, 165: 666-677.

[11] Sprodowski C, Morgenstern K. Temperature-dependent change of the fractal dimension of $\mathrm{Cu}$ dendrites on $\mathrm{Cu}$ (111). New Journal of Physics, 2020, 22(6): 1-8.

[12] Shen Y Z, Liu J H, Yang S F, et al. Dendrite growth behavior in directionally solidified Fe-C-Mn-Al alloys. Journal of Crystal Growth, 2019, 511: 118-126.

[13] Hu X P, Zhao Y, Wang Q, et al. Effects of process parameters on semi-solid squeeze casting performance of aluminum alloy scrolls for scroll compressors. China Foundry, 2020, 17(5): 347-356.

[14] Guo E Y, Shuai S S, Kazantsev D, et al. The influence of nanoparticles on dendritic grain growth in $\mathrm{Mg}$ alloys. Acta Materialia, 2018, 152: 127-137.

[15] Du J L, Zhang A, Guo Z P, et al. Atomistic underpinnings for growth direction and pattern formation of hcp magnesium alloy dendrite. Acta Materialia, 2018, 161: 35-46.

[16] Pettersen K, Lohne O, Ryum N. Dendritic solidification of magnesium alloy AZ91. Metallurgical Transactions A, 1990, 21: 221-230.

[17] Shuai S S, Guo E Y, Wang J, et al. Synchrotron tomographic quantification of the influence of $\mathrm{Zn}$ concentration on dendritic growth in Mg-Zn alloys. Acta Materialia, 2018, 156: 287-296.

[18] Wang M Y, Williams J J, Jiang L, et al. Dendritic morphology of $\alpha-M g$ during the solidification of Mg-based alloys: 3D experimental characterization by X-ray synchrotron tomography and phase-field simulations. Scripta Materialia, 2011, 65(10): 855-858.

[19] Yang M, Xiong S M, Guo Z P. Effect of different solute additions on dendrite morphology and orientation selection in cast binary magnesium alloys. Acta Materialia, 2016, 112: 261-272.

[20] Wu M W, Hua L, Xiong S M. Modeling studies on divorced eutectic formation of high pressure die cast magnesium alloy. China Foundry, 2018, 15(1): 58-65.

[21] Han G M, Han Z Q, Luo A A, et al. A phase field model for simulating the precipitation of multi-variant beta- $\mathrm{Mg}_{17} \mathrm{Al}_{12}$ in $\mathrm{Mg}$ Al-based alloys. Scripta Materialia, 2013, 68(9): 691-694.

[22] Zhu M F, Stefanescu D M. Virtual front tracking model for the quantitative modeling of dendritic growth in solidification of alloys. Acta Materialia, 2007, 55(5): 1741-1755.

[23] Pineau A, Guillemot G, Tourret D, et al. Growth competition between columnar dendritic grains-cellular automaton versus phase field modeling. Acta Materialia, 2018, 155: 286-301.

[24] Yu A S, Yang X J, Guo H M. Phase field lattice Boltzmann model for non-dendritic structure formation in aluminum alloy from LSPSF machine. Transactions of Nonferrous Metals Society of China, 2020, 30(3): 559-570.
[25] Feng L, Lu N N, Gao Y L, et al. Phase-field simulation of secondary dendrite growth in directional solidification of binary alloys. China Foundry, 2019, 16(2): 97-104.

[26] Zhang A, Du J L, Zhang X P, et al. Phase-field modeling of microstructure evolution in the presence of bubble during solidification. Metallurgical and Materials Transactions A, 2020, 51: 1023-1037.

[27] Wang S, Zaeem M A, Horstemeyer M F, et al. Investigating thermal effects on morphological evolution during crystallisation of hcp metals: Three-dimensional phase field study. Materials Technology, 2012, 27(5): 355-363.

[28] Wang Y B, Peng L M, Ji Y Z, et al. Effect of cooling rates on the dendritic morphology transition of Mg-6Gd alloy by in situ X-ray radiography. Journal of Materials Science and Technology, 2018, 34(7): 1142-1148.

[29] Zhang X, Wang Y, Liu D R, et al. Effect of stirring rate on grain morphology of Mg-Al alloy semi-solid structure by phase field lattice Boltzmann simulation. Journal of Crystal Growth, 2020, 543: 125704

[30] Pan H W, Han Z Q, Liu B C. Study on dendritic growth in pressurized solidification of Mg-Al alloy using phase field simulation. Journal of Materials Science and Technology, 2016, 32(1): 68-75.

[31] Yang M, Xiong S M, Guo Z P. Characterisation of the 3-D dendrite morphology of magnesium alloys using synchrotron X-ray tomography and 3-D phase-field modelling. Acta Materialia, 2015, 92: 8-17.

[32] Wang M Y, Jing T, Liu B C. Phase-field simulations of dendrite morphologies and selected evolution of primary $\alpha-M g$ phases during the solidification of Mg-rich Mg-Al-based alloys. Scripta Materialia, 2009, 61(8): 777-780.

[33] Eiken J. Phase-field simulation of microstructure formation in technical magnesium alloys. International Journal of Materials Research, 2010, 101(4): 503-509.

[34] Ramirez J C, Beckermann C, Karma A, et al. Phase-field modeling of binary alloy solidification with coupled heat and solute diffusion. Physical Review E, 2004, 69(5): 051607.

[35] Guo Z P, Mi J, Grant P S. An implicit parallel multigrid computing scheme to solve coupled thermal-solute phase-field equations for dendrite evolution. Journal of Computational Physics, 2012, 231(4): 1781-1796.

[36] Zhang X, Kang J, Guo Z P, et al. Development of a Para-AMR algorithm for simulating dendrite growth under convection using a phase-field-lattice Boltzmann method. Computer Physics Communications, 2018, 223: 18-27.

[37] Guo Z P, Mi J, Xiong S M, et al. Phase field study of the tip operating state of a freely growing dendrite against convection using a novel parallel multigrid approach. Journal of Computational Physics, 2014, 257: 278-297.

[38] Guo Z P, Xiong S M. On solving the 3-D phase field equations by employing a parallel-adaptive mesh refinement (Para-AMR) algorithm. Computer Physics Communications, 2015, 190: 89-97.

[39] Flemings M C. Solidification processing. New York: McGraw-Hill, 1974.

[40] Chalmers B. Principles of solidification. New York: Wiley, 1966.

[41] Kurz W, Fisher D J. Fundamentals of solidification. Switzerland: Trans. Tech. Publications, 1986.

[42] Lipton J, Kurz W, Trivedi R. Rapid dendrite growth in undercooled alloys. Acta Metallurgica, 1987, 35(4): 957-964. 